\title{
Ultrasonographic Evaluation of Placental Thickness in Normal Singleton Pregnancies for Estimation of Gestation Age
}

\author{
Ridhi Adhikari, Pravin Kumar Deka, Ashok Tayal, Pramod Kumar Chettri \\ Department of Radiodiagnosis, College of Medical Sciences \& Teaching Hospital, Bharatpur, Nepal
}

Email address:

ridhi.adhikari@gmail.com (R. Adhikari)

\section{To cite this article:}

Ridhi Adhikari, Pravin Kumar Deka, Ashok Tayal, Pramod Kumar Chettri. Ultrasonographic Evaluation of Placental Thickness in Normal Singleton Pregnancies for Estimation of Gestation Age. International Journal of Medical Imaging. Vol. 3, No. 6, 2015, pp. $143-147$. doi: $10.11648 /$ j.jimi.20150306.16

\begin{abstract}
Background: Use of ultrasonography is the most effective method to estimate the gestational age. Placental thickness can be used as a new parameter to estimate the gestational age of the fetus and to assess the growth pattern of the placenta with advancing gestational age. Methods: This was a prospective cross sectional study consisting 150 normal antenatal women who were assessed clinically at the Department of Obstetrics and Gyaenocology and referred to the Department of Radiology, College of Medical Sciences, Bharatpur for ultrasonography. Antenatal women of all gestational ages from 11 weeks to 40 weeks of gestation with normal singleton pregnancies were included in the study. Placental thickness value, in $\mathrm{mm}$, was calculated by averaging the three best measurements for each case. Relationship of placental thickness (in $\mathrm{mm}$ ), measured at the level of insertion of umbilical cord with advancing gestational age in weeks was studied. Correlation of mean placental thickness with gestational age from 11 weeks to 40 weeks was calculated. Results: It was observed that the placental thickness gradually increased from approximately $11 \mathrm{~mm}$ at 11 weeks to $38.33 \mathrm{~mm}$ at 40 weeks of gestation. From 11 to 34 weeks of gestation, the placental thickness (in $\mathrm{mm}$ ) almost matched the gestational age in weeks, thereafter from 35 to 40 weeks; the placental thickness was lower by 1 to $2 \mathrm{~mm}$. Conclusion: The relationship between the placental thickness and gestational age was linear and direct. Placental thickness (in $\mathrm{mm}$ ) measurement can be an important additional parameter for estimating gestational age along with other parameters especially from 11 to 34 weeks of gestation.
\end{abstract}

Keywords: Gestational Age, Placenta, Ultrasonography, Umbilical Cord

\section{Introduction}

Accurate assessment of gestational age is an important part of any obstetric examination and presently the most effective way to date pregnancy is by the use of ultrasonography. Several sonographically derived fetal parameters are used to date pregnancy. They are fetal crown-rump length (CRL), biparietal diameter (BPD), head circumference (HC), femur length (FL) and abdominal circumference (AC). However, there are some drawbacks in these parameters in estimating the gestational age. So, there is a need of another parameter for supplementing the gestational age estimation with minimal error.

Placental evaluation by ultrasonography has been used to characterize placental position and morphologic changes as the placenta matures. [1,2] The role of ultrasonography in the evaluation of morphology and detection of placental abnormalities in entities such as non-immune hydrops, gestational diabetes and intrauterine growth retardation (IUGR) has been well established. One additional ultrasonographic parameter used to assess the placenta is placental thickness. The measurement of placental thickness is relatively simple and clinically useful. Abnormal thickness of placenta is well recognized as a diagnostic harbinger in a wide spectrum of pathologic events. Placental thickness can contribute to the management of fetus at risk. [3] Few authors have studied the role of placental thickness as a new parameter for estimating gestational age and placental thickness nomograms in relation to gestational age have been published. [4-6].

The purpose of the present study of measuring placental thickness at the level of umbilical cord insertion site was to assess the relationship of placental thickness with gestational 
age and also to assess the growth pattern of placenta with advancing gestational age.

\section{Material and Methods}

This was a prospective cross sectional study consisting 150 normal antenatal women who were assessed clinically at the Department of Obstetrics and Gyaenocology and referred to the Department of Radiology, College of Medical Sciences, Bharatpur for ultrasonography, during the study period from September 2011 to August 2013.

After taking a detailed history, the antenatal women were examined for placental thickness, gestational age and fetal weight ultrasonographically. Subjects were included in the study if they meet the inclusion criteria. Written informed consent was obtained in all subjects before the ultrasonographic examination. The normal antenatal women of all gestational ages from 11 weeks to 40 weeks of gestation referred to the department of Radiology, College of Medical Sciences, Bharatpur for routine antenatal ultrasound were included in the study. Patients with pregnancy induced hypertension, diabetes mellitus, intrauterine growth retardation, hydrops fetalis, congenital malformations and twins were excluded.

The grey scale real time ultrasonographic examinations were performed using a TOSHIBA XARIO ultrasound equipment system. The transducer of the TOSHIBA XARIO ultrasound used with a scanner frequency of $3.5 \mathrm{MHz}$. Subjects were scanned with a moderately distended bladder in supine position. The transducer was placed on the skin surface after applying the coupling agent. Placental thickness, in $\mathrm{mm}$, was measured at the level of cord insertion site. The transducer was oriented to scan perpendicular to both the chorionic and basal plates, as tangential scan will distort the measurement of the thickness of placenta. All placental measurements were taken during the relaxed phase of the uterus as contractions can spuriously increase the placental thickness.

\section{Statistical Analysis}

The statistical software Statistical Package for Social Sciences (SPSS) 20.0 was used for the analysis of the data and Microsoft word and Excel 10.0 have been used to generate graphs and tables. The mean values of placental thickness, in $\mathrm{mm}$, along with respective standard deviation (SD) were computed for each gestational age from 11 weeks to 40 weeks. The $95 \%$ Confidence Interval were also calculated. Pearson's correlation analysis was also used to establish the degree of relationship between placental thickness, gestational age, BPD, FL and AC. Values were expressed as mean $\pm \mathrm{SD}$. Statistical tests were two-tailed with $\mathrm{p}<0.01$ to indicate statistical significance.

\section{Results}

In the total study group of 150 normal antenatal women, the age ranged between 17 years to 35 years. The mean age was 22.64 years and a SD of 3.30. The distribution of cases with respect to maternal age groups is given in Table 1 . Majority of antenatal women were in the age group between 20 and 30 years.

Table 1. Maternal age distribution as declared by the subjects at the time of examination.

\begin{tabular}{lll}
\hline Age group & Number of women $(\mathbf{n}=\mathbf{1 5 0})$ & Percentage \\
\hline$<20$ Years & 41 & $27.3 \%$ \\
$20-30$ Years & 107 & $71.3 \%$ \\
$>30$ Years & 2 & $1.3 \%$ \\
\hline
\end{tabular}

The minimum gestational age was 11.57 weeks and the maximum gestational age was 40.00 weeks with a mean gestational age of 25.49 weeks and a SD of 8.00. Table 2.

Table 2. Gestational age at the time of examination.

\begin{tabular}{lll}
\hline Gestational age (trimester) & Number of women $(\mathbf{n = 1 5 0 )}$ & Percentage \\
\hline First Trimester & 3 & $2.0 \%$ \\
Second Trimester & 81 & $54.0 \%$ \\
Third Trimester & 66 & $44.0 \%$ \\
\hline
\end{tabular}

Out of 150 normal antenatal women studied, anterior placenta was noted in $36 \%$, posterior in $46 \%$, fundal in $11 \%$ and lateral in $7 \%$ cases. Distribution of placental position is shown in Table 3.

Table 3. Distribution of placental position at the time of examination.

\begin{tabular}{lll}
\hline Placental position & Number of women $(\mathbf{n = 1 5 0})$ & Percentage \\
\hline Anterior & 54 & $36.0 \%$ \\
Posterior & 69 & $46.0 \%$ \\
Fundal & 16 & $11.0 \%$ \\
Lateral & 11 & $7.0 \%$ \\
\hline
\end{tabular}

Placental grading is shown in Table 4. Grade 1 placenta was present in 93 subjects, 37 had grade 2 and 20 had grade 3 placenta.

Table 4. Placental grade according to the maturity of placenta as seen on ultrasonography.

\begin{tabular}{lll}
\hline Placental grade & Number of women $(\mathbf{n}=\mathbf{1 5 0})$ & Percentage \\
\hline Grade 1 & 93 & $62.0 \%$ \\
Grade 2 & 37 & $24.7 \%$ \\
Grade 3 & 20 & $13.3 \%$ \\
\hline
\end{tabular}

The minimum placental thickness was $11.00 \mathrm{~mm}$, the maximum placental thickness was $38.33 \mathrm{~mm}$ with a mean placental thickness of $25.21 \mathrm{~mm}$ and a SD of 7.80. Results of measurements of placental thickness at each week of gestational age from 11 to 40 weeks are shown in Table 5. It was observed that the placental thickness gradually increased from approximately $11.00 \mathrm{~mm}$ at 11 weeks to $38.33 \mathrm{~mm}$ at 40 weeks of gestation. From 11 to 34 weeks of gestation, the placental thickness (in $\mathrm{mm}$ ) almost matched the gestational age in weeks, thereafter from 35 to 40 weeks; the placental thickness was lower by 1 to $2 \mathrm{~mm}$. At no stage of pregnancy was the normal placenta greater than $39 \mathrm{~mm}$. 
Table 5. Effect of advancing gestational age on placental thickness.

\begin{tabular}{|c|c|c|c|}
\hline $\begin{array}{l}\text { Gestational age (weeks) according to Hadlock } \\
\text { formula [7] }\end{array}$ & Number of cases & $\begin{array}{l}\text { Placental thickness in mm (mean } \\
\pm \text { SD) }\end{array}$ & $\begin{array}{l}\text { 95\% Confidence interval (Lower- } \\
\text { Upper) }\end{array}$ \\
\hline 11 & 1 & $11.00 \pm 00$ & - \\
\hline 12 & 2 & $12.00 \pm 00$ & - \\
\hline 13 & 6 & $13.55 \pm 0.80$ & $12.91-14.19$ \\
\hline 14 & 3 & $13.89 \pm 0.19$ & $13.67-14.11$ \\
\hline 15 & 7 & $14.95 \pm 0.30$ & $14.73-15.17$ \\
\hline 16 & 9 & $17.92 \pm 4.94$ & $14.69-21.15$ \\
\hline 17 & 4 & $17.08 \pm 0.16$ & $16.92-17.24$ \\
\hline 18 & 11 & $18.39 \pm 0.62$ & $19.02-19.76$ \\
\hline 19 & 6 & $18.83 \pm 0.91$ & $18.12-19.56$ \\
\hline 20 & 8 & $20.25 \pm 0.61$ & $19.83-20.67$ \\
\hline 21 & 4 & $21.25 \pm 0.32$ & $20.94-21.56$ \\
\hline 22 & 4 & $21.83 \pm 0.19$ & $21.64-22.02$ \\
\hline 23 & 3 & $22.78 \pm 0.69$ & $22.00-23.56$ \\
\hline 24 & 5 & $24.53 \pm 0.69$ & $23.93-25.13$ \\
\hline 25 & 3 & $25.00 \pm 00$ & - \\
\hline 26 & 5 & $26.07 \pm 0.15$ & $25.94-26.20$ \\
\hline 27 & 0 & - & - \\
\hline 28 & 5 & $28.27 \pm 0.59$ & $27.75-28.79$ \\
\hline 29 & 3 & $29.22 \pm 0.69$ & $28.44-30.00$ \\
\hline 30 & 6 & $30.22 \pm 0.40$ & $29.90-30.54$ \\
\hline 31 & 4 & $31.00 \pm 00$ & - \\
\hline 32 & 9 & $32.33 \pm 0.98$ & $31.69-32.97$ \\
\hline 33 & 9 & $32.63 \pm 0.98$ & $31.99-33.27$ \\
\hline 34 & 12 & $34.05 \pm 0.66$ & $33.68-34.42$ \\
\hline 35 & 6 & $34.72 \pm 1.02$ & $33.92-35.54$ \\
\hline 36 & 6 & $35.72 \pm 1.10$ & $34.84-36.60$ \\
\hline 37 & 4 & $35.67 \pm 0.94$ & $34.75-36.59$ \\
\hline 38 & 2 & $36.16 \pm 1.64$ & $33.89-38.43$ \\
\hline 39 & 0 & - & - \\
\hline 40 & 1 & $38.33 \pm 00$ & - \\
\hline
\end{tabular}
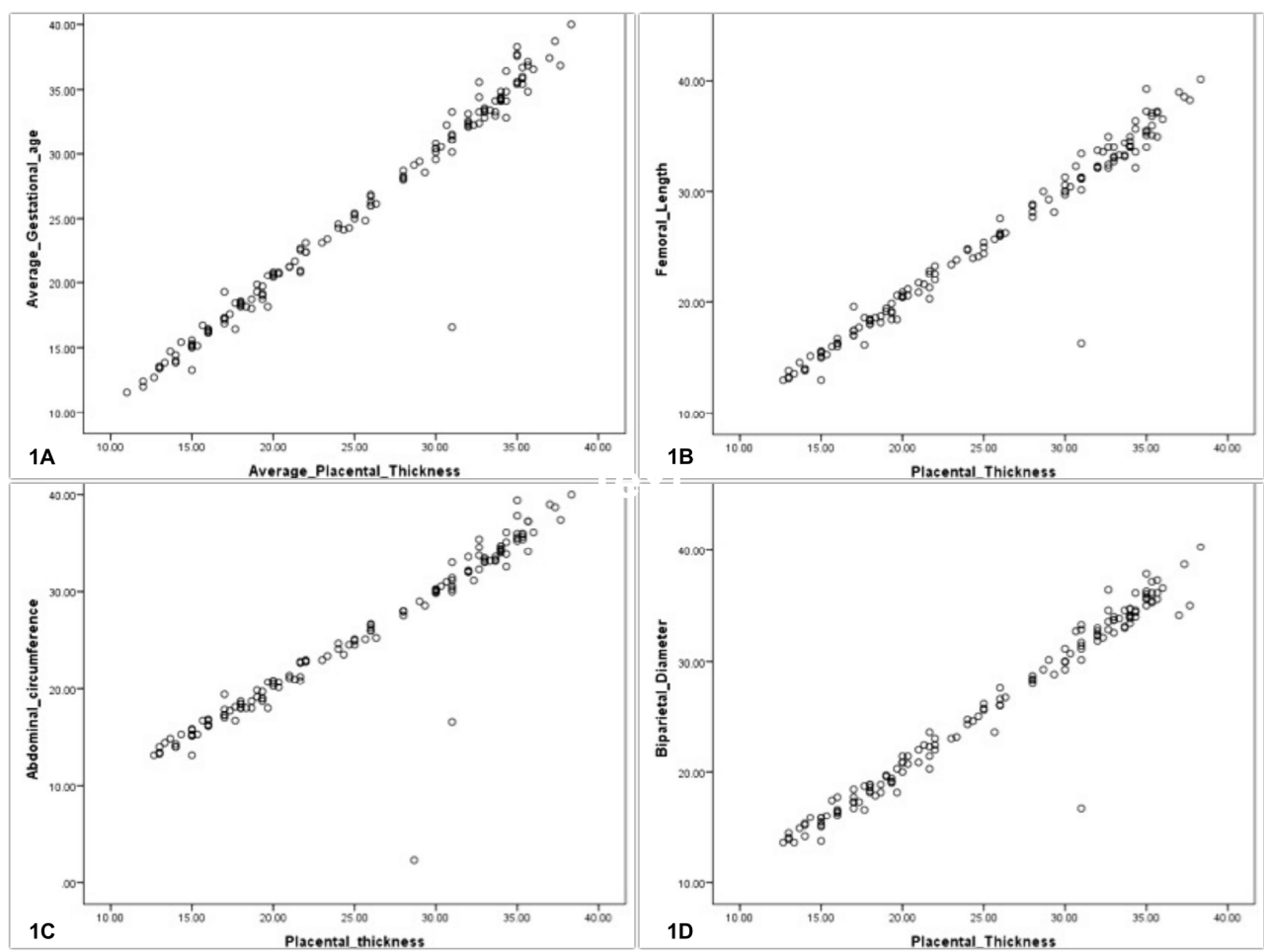

Figures 1. 1A. Correlation graph between average placental thickness (in mm) and gestational age (in weeks) ( $n=150$ ) 1B. Graph of femoral length (in weeks) against placental thickness (mm). 1C. Graph of abdominal circumference (in weeks) against placental thickness (in mm). 1D. Graph of biparietal diameter (in weeks) against placental thickness (in $\mathrm{mm}$ ). 
To prove that there was a correlation the between placental thickness and the gestational age, the correlation coefficient was calculated. In the second trimester, the Pearson correlation coefficient ' $r$ ' between placental thickness and gestational age was 0.914 and $p$ value of $<0.001$ which was significant at 0.01 level. In the third trimester, the Pearson correlation coefficient ' $r$ ' between placental thickness and gestational age was 0.946 and $p$ value of $<0.001$ which was significant at 0.01 level. Pearson's correlation values between placental thickness and gestational age are shown in Fig. $1 \mathrm{~A}$.

There was a significant positive correlation between placental thickness and, FL, BPD and AC in the second and third trimesters; with all parameters having identical relationships with placental thickness. The Pearson Correlation coefficient ' $r$ ' between placental thickness and FL was 0.982 and $p$ value of $<0.001$ which was significant at 0.01 level. The Pearson Correlation coefficient ' $r$ ' between placental thickness and AC was 0.944 and $p$ value of $<0.001$ which was significant at 0.01 level. The Pearson Correlation coefficient ' $\mathrm{r}$ ' between placental thickness and BPD was 0.981 and $\mathrm{p}$ value of $<0.001$ which was significant at 0.01 level. (Fig. 1B, 1C, 1D).

\section{Discussion}

The present study assessed the relationship of placental thickness (in $\mathrm{mm}$ ) with ultrasonographic gestational age (in weeks) and also the growth pattern with advancing gestational age. The study showed that the placental thickness (in $\mathrm{mm}$ ) increases steadily with increasing gestational age (in weeks) in a linear fashion and almost matching the gestational age from 11 to 35 weeks of gestation. The rate of increase of placental thickness gradually diminished from 36 to 40 weeks and was less by 1 to $2 \mathrm{~mm}$ compared to gestational age (in weeks). To prove that there was a correlation between placental thickness and the gestational age, the correlation coefficient was calculated and it was found to be $r=0.914, r=0.946$ and $r=0.984$ for the $2 \mathrm{nd}, 3 \mathrm{rd}$ and average trimesters respectively and the $\mathrm{p}$ value was $<0.001$, thereby establishing a positive correlation between the two variables.

The results of the present study are consistent with the observations made by authors of previous studies. Nagwani et al.[8] found average placental thickness to be roughly equivalent to gestational age (in weeks). They reported that the mean thickness of the placenta was $3.90 \pm 1.1 \mathrm{~cm}$ which increased till 38 weeks of gestation, thereafter decreased. Mital et al.[4] also found an increasing trend in the values of mean placental thickness (in $\mathrm{mm}$ ) with increase in gestational age (in weeks) and the placental thickness (in mm) coincides almost exactly with the gestational age in weeks. Jain et al. [5] reported similar correlations between placental thickness and gestational age. They found placental thickness (in mm) almost matched gestational age (in weeks) from 27 weeks to 33 weeks of gestation. Baghel et al.[9] reported that placental thickness in millimeters almost coincides with gestational age in weeks at 24 weeks (24.5 mm at 24 weeks), 32 (31.8 $\mathrm{mm}$ at 32 weeks) and 36 weeks ( $35.5 \mathrm{~mm}$ at 36 weeks). Nyberg and Finberg [10] also reported that as a rule of thumb, placental thickness parallels gestational age (in weeks). Similarly in a recent study by Karthikeyan et al.[11] had reported that placental thickness can be used as a predictor of the gestational age, in the women in whom the LMP is unreliable or is not known.

Fetal growth parameters such as BPD, and AC are used in the sonographic estimation of gestational age and weight of the fetus in the second and third trimesters. [12] It is a standard practice to assess FL as part of the evaluation of fetal size and morphology. FL has been established as an accurate parameter for estimating gestational age in the second and third trimesters. [12] Present study showed a statistically significant positive correlation between placental thickness, FL, BPD and AC. This means that estimated fetal weight which is calculated based on a formula having FL, $\mathrm{BPD}$ and $\mathrm{AC}$ as variables [13] is dependent on placental thickness.

\section{Limitations}

In this study we adopted a cross sectional design, which is made up of observations on different individuals and did not follow the subjects longitudinally. So, it may not provide a clear understanding in individual growth patterns. [14] However, it is a reasonable approximation of a true placental growth curve. There is a need to recruit subjects at the beginning of the second trimester and scan them at regular intervals to ascertain the relationship between placental thickness and growth parameters. Such a study should be multiple to increase the validity of the result. In our environment this will be a difficult task because of low literacy level and a trend of home delivery.

Accuracy of placental measurements depends on making a perpendicular scan of the placenta and care should be taken in acquisition and interpretation of the images to prevent spurious measurements. Placental thickness measurement using 3D ultrasonography may more accurately assess placental size than placental thickness measurements. However, 3D ultrasonography is expensive, time consuming and not widely available. The parameter of placental thickness may vary among different population groups. Population specific nomograms may be needed derived from large sample sizes. The placental growth curves may be different for different population groups. Short placental insertion site may spuriously suggest placental thickening in a normal placenta. Moreover, cord insertion site on the placenta was difficult to image in normal term pregnancies, especially in posterior locations.

\section{Conclusion}

Sonographic measurement of placental thickness, at the level of cord insertion site, is relatively simple and is clinically 
useful. Placental thickness (in $\mathrm{mm}$ ) increases in a linear fashion with advancing gestational age (in weeks) and almost matching it from 11 to 35 weeks of gestation. Moreover, there was a correlation between placental thickness and the gestational age. It can be an additional indicator of estimating gestational age especially where the duration of pregnancy is unknown or uncertain. It was observed that the relationship of placental thickness with gestational age falls marginally and the rate of growth of placental thickness decreased after 36 weeks of gestation. However, a large series of longitudinal study in the future could have been much useful for estimation of fetal maturity and assessment of gestational age.

\section{Authors' Contribution}

RA conceived the concept and wrote the first draft. RA performed the sonographic investigations. RA, PKD, AT and $\mathrm{PKC}$ involved in the data collection, critically reviewed the manuscript and all approved content of the final manuscript.

\section{References}

[1] Kobayashi M, Hellman LM, Fillisti L. Placental localization by ultrasound. Am J Obstet Gynecol. 1970; 106: 279-85.

[2] Gottesfield KR, Thompson HE, Holmes JH, Taylor ES. Ultrasonic placentography - a new method of placental localization. Am J Obstet Gynecol. 1966; 96: 538-47.

[3] Jauniaux E. Placental ultrasonographic measurements: What can we learn and is it worth doing routinely? Ultrasound Obstet Gynecol. 1992; 2: 241-2.

[4] Mital P, Hooja N, Mehndiratta K. Placental thickness: a sonographic parameter for estimating gestational age of the fetus. Indian J Radiol Imaging. 2002; 12: 553-4.

[5] Jain A, Kumar G, Agarwal U, Kharakwal S. Placental thickness -a sonographic indicator of gestational age. J Obstet Gynaecol Ind. 2001; 51: 48-9.
[6] Tanawattancharoen S, Manotaya S, Uerpairojkit B. Ultrasound measurement of placental thickness in normal singleton pregnancy. Thai J Obstet Gynecol. 2000; 10: 73-8.

[7] Hadlock FP, Deter RL, Harrist RB, Park SK. Estimating foetal age: computer-assisted analysis of multiple foetal growth parameters. Radiology. 1984; 152: 497-501.

[8] Nagwani M, Sharma PK, Singh U, Rani A, Mehrotra S. Ultrasonographic measurement of placental thickness and its correlation with gestational age - a cross-sectional ultrasonographic study. International Journal of Advanced Research. 2014; 2: 354-360.

[9] Baghel P, Bahel V, Paramhans R, Sachdev P, Onkar S. Correlation of placental thickness estimated by ultrasonography with gestational age and fetal outcome. Indian Journal of Neonatal Medicine and Research. 2015; 3: 19-24.

[10] Nyberg DA, Finberg HJ. The placenta, placental membranes and umbilical cord. In: Newburgh DA, Mahony BS, Pretorius DH, editors. Diagnostic ultrasound of foetal anomalies. St. Louis: Mosby year book publishers; 1990. p. 623-75.

[11] Karthikeyan T, Subramaniam RK, Johnson W, Prabhu K. Placenatal thickness and its correlation to gestational age and foetal growth parameters- a cross sectional ultrasonographic study. J Clin Diagn Res. 2012; 6: 1732-5.

[12] Benson CB, Doubilet PM. Foetal measurements: normal and abnormal foetal growth. In: Rumak $\mathrm{CM}$, Wilson SR, Charboneau JW, Levine D, editors. Diagnostic ultrasound, 4th ed. Philadelphia: Elsevier; 2011. p. 1455-71.

[13] Shepard MJ, Richards VA, Berkowitz RL, Warsof SL, Hobbins JC. An evaluation of two equations for predicting foetal weight by ultrasound. Am J Obstet Gynecol. 1982; 142: $47-54$

[14] Wolf H, Oosting H, Treffers PE. A longitudinal study of relationship between placental and foetal growth as measured by ultrasonography. Am J Obstet Gynecol. 1989; 161: 1140-5. 